Article

\title{
Monocyte Chemotactic Protein-1 as a Potential Biomarker for Early Anti-Thrombotic Therapy after Ischemic Stroke
}

\author{
Hans Worthmann ${ }^{1}$, Reinhard Dengler ${ }^{1}$, Helmut Schumacher ${ }^{2}$, Andreas Schwartz ${ }^{3}$, \\ Wolfgang G. Eisert ${ }^{1,4}$, Ralf Lichtinghagen ${ }^{5, \dagger}$ and Karin Weissenborn ${ }^{1, \dagger, *}$
}

1 Department of Neurology, Hannover Medical School, Carl-Neuberg-Str. 1, Hannover 30625, Germany; E-Mails: worthmann.hans@mh-hannover.de (H.W.); dengler.reinhard@mh-hannover.de (R.D.); eisert.wolfgang@mh-hannover.de (W.G.E.) Boehringer Ingelheim Pharma GmbH \& Co. KG, Binger Str. 173, Ingelheim 55216, Germany; E-Mail: helmut.schumacher@boehringer-ingelheim.com

3 Department of Neurology, Nordstadt Klinikum Hannover, Haltenhoffstr. 41, Hannover 30167, Germany; E-Mail: andreas.schwartz@krh.eu

4 Institute of Biophysics, University of Hannover, Herrenhäuserstr. 2, Hannover 30419, Germany

5 Department of Clinical Chemistry, Hannover Medical School, Carl-Neuberg-Str. 1, Hannover 30625, Germany; E-Mail: lichtinghagen.ralf@mh-hannover.de

$\dagger$ These authors contributed equally to this work.

* Author to whom correspondence should be addressed; E-Mail: weissenborn.karin@mh-hannover.de; Tel.: +49-511-532-2339; Fax: +49-511-532-3115.

Received: 7 May 2012; in revised form: 21 June 2012 / Accepted: 3 July 2012 /

Published: 12 July 2012

Abstract: Inflammation following ischemic brain injury is correlated with adverse outcome. Preclinical studies indicate that treatment with acetylsalicylic acid + extended-release dipyridamole (ASA + ER-DP) has anti-inflammatory and thereby neuroprotective effects by inhibition of monocyte chemotactic protein-1 (MCP-1) expression. We hypothesized that early treatment with ASA + ER-DP will reduce levels of MCP-1 also in patients with ischemic stroke. The EARLY trial randomized patients with ischemic stroke or TIA to either ASA + ER-DP treatment or ASA monotherapy within $24 \mathrm{~h}$ following the event. After 7 days, all patients were treated for up to 90 days with ASA + ER-DP. MCP-1 was determined from blood samples taken from 425 patients on admission and day 8 . The change in MCP-1 from admission to day 8 did not differ between patients treated with ASA + ER-DP and ASA monotherapy $(p>0.05)$. Comparisons within MCP-1 baseline 
quartiles indicated that patients in the highest quartile ( $>217-973 \mathrm{pg} / \mathrm{mL})$ showed improved outcome at 90 days if treated with ASA + ER-DP in comparison to treatment with ASA alone $(p=0.004)$. Our data does not provide any evidence that treatment with ASA + ER-DP lowers MCP-1 in acute stroke patients. However, MCP-1 may be a useful biomarker for deciding on early stroke therapy, as patients with high MCP-1 at baseline appear to benefit from early treatment with ASA + ER-DP.

Keywords: ischemic stroke; monocyte chemoattractant protein-1 (MCP-1); antithrombotic therapy; neuroprotection; dipyridamole; acetylsalicylic acid (ASA)

\section{Introduction}

Considering recent findings in experimental animals and stroke patients, the extent of the secondary inflammatory response that immediately follows ischemic brain injury, is suggested to be significantly accountable for the outcome in ischemic stroke [1-5]. The chemokine monocyte chemotactic protein-1 (MCP-1) attracts leukocytes to ischemic tissue [6]. These activated leukocytes accumulate in the cerebral microvessels and trigger the inflammatory reaction by further release of cytokines, oxygen-free radicals and proteolytic enzymes like matrix-metalloproteinases [7].

Former preclinical and clinical studies have suggested anti-inflammatory properties of dipyridamole, such as scavenging of active oxygen metabolites, reduction of the TNF-alpha and IL-8 concentration and inhibition of the adhesion of neutrophils to vascular endothelium in acute ischemic stroke patients [8-13]. In cell cultures of human leukocytes and platelets the addition of dipyridamole inhibited the expression of MCP-1 [8].

We hypothesized that early treatment with ASA + ER-DP (acetylsalicylic acid + extended-release dipyridamole) in patients with ischemic stroke or TIA will result in reduced levels of MCP-1.

\section{Results}

\subsection{Study Population}

A total of 425 randomized patients (220 patients assigned to early ASA + ER-DP and 205 patients to early ASA alone) with TIA/stroke (median age 68 years; median NIHSS at admission 3) were included in this sub-study of the EARLY trial. Both treatment groups were well balanced except for a higher number of current smokers in the early ASA + ER-DP group (Table 1).

Table 1. Demographics and baseline characteristics.

\begin{tabular}{lccc}
\hline & Total $(\boldsymbol{n}=\mathbf{4 2 5})$ & $\begin{array}{c}\text { Early ASA + ER-DP } \\
(\boldsymbol{n}=\mathbf{2 2 0})\end{array}$ & Early ASA $(\boldsymbol{n}=\mathbf{2 0 5})$ \\
\hline Age (years) $\geq 65$ & $68(27-95)$ & $67.0(27-95)$ & $69.0(37-88)$ \\
& $272(64 \%)$ & $131(60 \%)$ & $141(69 \%)$ \\
Men & $272(64 \%)$ & $146(66 \%)$ & $126(62 \%)$ \\
White & $424(100 \%)$ & $219(100 \%)$ & $205(100 \%)$ \\
\hline
\end{tabular}


Table 1. Cont.

\begin{tabular}{lccc}
\hline BMI $\left(\mathrm{kg} / \mathrm{m}^{2}\right)$ & $27.4(4.0)$ & $27.4(4.1)$ & $27.3(4.0)$ \\
BMI $\geq 30$ & $103(24 \%)$ & $53(24 \%)$ & $50(24 \%)$ \\
\hline Smoking & & & $105(51 \%)$ \\
\hline Never & $198(47 \%)$ & $93(42 \%)$ & $61(30 \%)$ \\
Ex-smoker & $125(29 \%)$ & $64(29 \%)$ & $37(18 \%)$ \\
Current & $100(24 \%)$ & $63(29 \%)$ & $156(76 \%)$ \\
\hline Concomitant disease & & & $51(25 \%)$ \\
\hline Hypertension & $317(75 \%)$ & $161(73 \%)$ & $63(31 \%)$ \\
Diabetes & $102(24 \%)$ & $51(23 \%)$ & $7(3 \%)$ \\
Hyperlipidaemia & $142(33 \%)$ & $79(36 \%)$ & $13(6 \%)$ \\
Atrial flutter or fibrillation & $16(4 \%)$ & $9(4 \%)$ & $27(13 \%)$ \\
Congestive heart failure & $19(5 \%)$ & $6(3 \%)$ & $2(0-5)$ \\
History of prior stroke & $61(14 \%)$ & $34(16 \%)$ & $3(0-20)$ \\
mRS & $2(0-5)$ & $2(0-5)$ & \\
NIHSS & $3(0-20)$ & $3(0-15)$ & \\
\hline
\end{tabular}

Data are median (range), $n(\%)$, or mean (SD). BMI = body mass index.

\subsection{MCP-1 Levels after Differential Antithrombotic Treatment}

The change in levels of MCP-1 from admission to day 8 did not differ between patients treated with ASA + ER-DP and ASA monotherapy as analyzed for the total study population (see Table 2, n.s. in Wilcoxon-Mann Whitney test).

Table 2. Monocyte chemotactic protein-1 (MCP-1) at baseline and day 8 .

\begin{tabular}{ccccc}
\hline & Total $(\boldsymbol{n}=\mathbf{4 2 5})$ & $\begin{array}{c}\text { Early ASA + ER-DP } \\
(\boldsymbol{n}=\mathbf{2 2 0})\end{array}$ & $\begin{array}{c}\text { Early ASA } \\
(\boldsymbol{n}=\mathbf{2 0 5})\end{array}$ & $\boldsymbol{p}$ \\
\hline Baseline & $183(145-217)$ & $182(143-215)$ & $184(148-223)$ & - \\
Day 8 & $186(156-229)$ & $186(154-231)$ & $186(158-227)$ & - \\
Change from baseline & $8(-25-41)$ & $9(-21-41)$ & $7(-28-41)$ & n.s. \\
\hline
\end{tabular}

MCP-1 (pg/mL), data are median (interquartile range), Wilcoxon-Mann-Whitney test for changes from baseline.

\subsection{Outcome at 90 Days Dependent on Stroke Severity but not on MCP-1}

Changes of MCP-1 from admission to day 8 were not predictive for outcome at 90 days, whereas clinical symptoms at day 8 assessed by NIHSS had the strongest predictive value for outcome at day 90 assessed by $\mathrm{mRS}$ independent of treatment $(p<0.0001)$.

\subsection{Association of MCP-1 Baseline Levels, Anti-Thrombotic Therapy and Outcome at 90 Days}

Logistic regression analysis revealed a significant interaction between treatment and MCP-1 levels at baseline on favorable outcome at day $90(p=0.009)$, indicating that the effect of treatment was not consistent across all MCP-1 levels. Comparisons within MCP-1 baseline quartiles showed that patients in the highest quartile (>217-973 pg/mL) showed improved outcome at 90 days (mRS 0 and 1) if treated with ASA + ER-DP in comparison to treatment with ASA alone ( $p=0.004)$ (Figure 1a). This 
effect was observed across all three NIHSS groups at baseline (Figure 1a,b). No significant differences between treatment groups were identified if $\mathrm{MCP}-1$ levels were not in the uppermost quartile at baseline (in the first three quartiles the respective p-values were 0.093, 0.301 and 0.792).

Figure 1. (a) (b) Association of MCP-1 baseline levels, anti-thrombotic therapy as either ASA + ER-DP (acetylsalicylic acid + extended-release dipyridamole) or ASA monotherapy and percentage of favorable outcome at 90 days. Patients in the highest MCP-1 quartile showed improved outcome if treated with ASA + ER-DP in comparison to treatment with ASA alone ( $p=0.004$ as indicated by $*$, Figure 1a). This effect was observed across all three NIHSS groups at baseline (Figure 1b). The predicted probability of favorable outcome as well as upper and lower 95\% confidence limits are indicated. Baseline quartiles of MCP-1 (pg/mL): Q1: 50- $\leq 145$, Q2: >145- $\leq 183$, Q3: >183- $\leq 217$, Q4: >217-973.

(a) Association of MCP-1 at bascl:nc, antiplatelet therapy and outcome at $90 \mathrm{~d}$

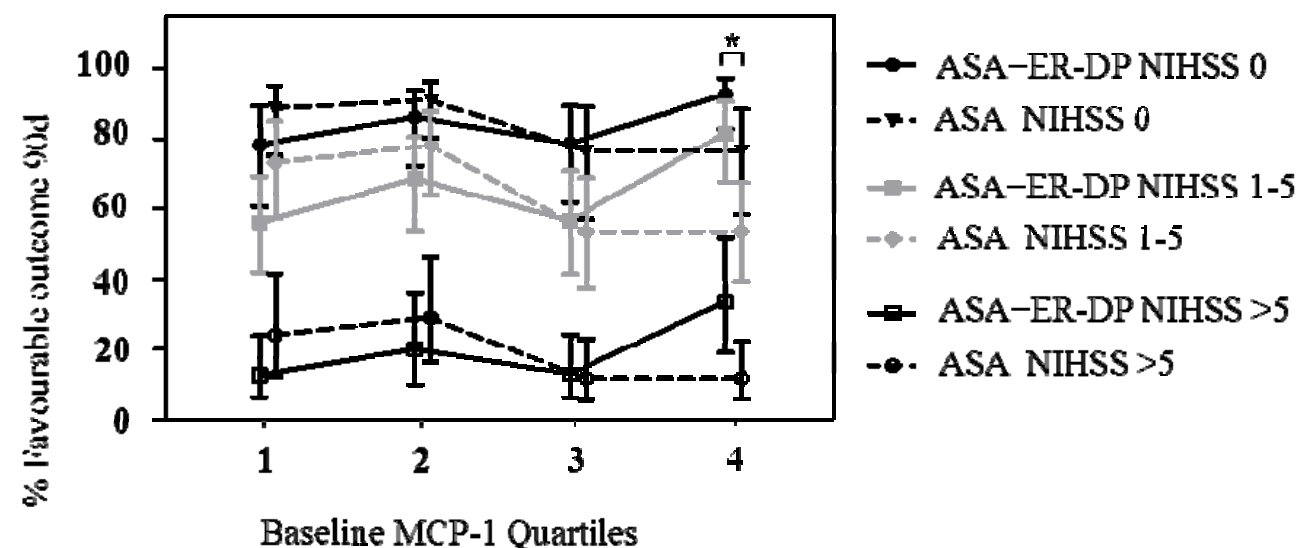

(b) High MCP-1 :evels at baseline (highest quartile) (n=107)

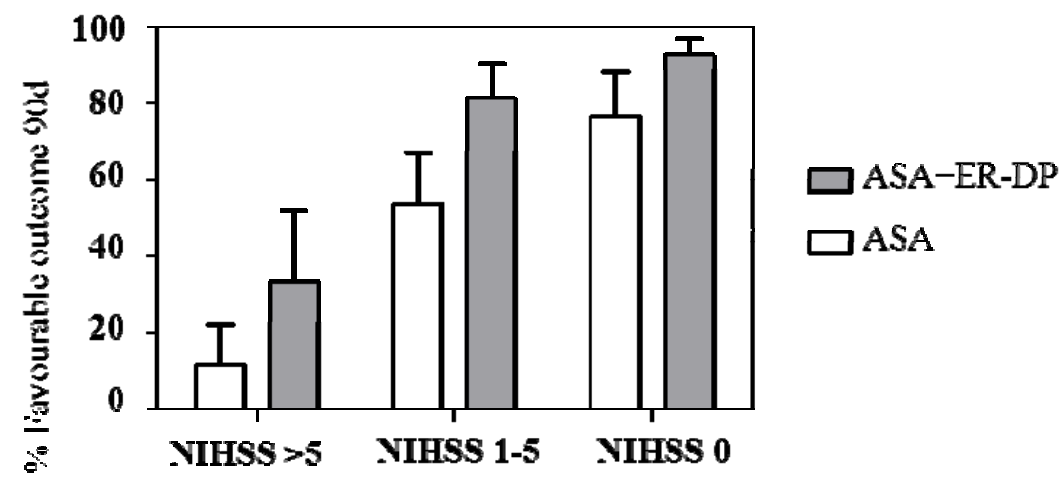

\section{Discussion}

Acute ischemia triggers an inflammatory response which is more than a bystander. The early inflammatory response contributes to the secondary progression of the ischemic lesion by increased expression and release of cytokines and chemokines [1-3]. Thus, MCP-1 has been shown to be rapidly increased within hours after the event $[3,14]$. This study investigated the effect of ASA + ER-DP versus 
ASA monotherapy upon the inflammatory response marker MCP-1 in 425 ischemic stroke patients of the EARLY study.

In vitro experiments showed a direct inhibition of $\mathrm{MCP}-1$ expression by dipyridamole either alone or in combination with ASA [8]. In contrast to this, our data did not show any differences for MCP-1 levels at day 8 or the change in MCP-1 levels from day 1 to day 8 between the treatment groups (Table 2). Nevertheless, our data showed that MCP-1 may be a useful biomarker for deciding on early stroke therapy as patients with MCP-1 levels in the uppermost baseline quartile did benefit more from early treatment with ASA + ER-DP compared to ASA monotherapy. Correlation of MCP-1 levels or change with clinical outcome was weak for lower MCP-1 plasma levels. Importantly, this effect seems to be independent of stroke severity at baseline (Figure 1a,b).

MCP-1 has repeatedly been suggested to play a pathogenic role in ischemic stroke. It has been considered as one of the key proteins for monocyte recruitment in inflammatory settings [6,15]. Smaller infarcts were observed for example after permanent middle cerebral artery occlusion in a mouse strain deficient in MCP-1 [16]. Recently Strecker et al. [17] suggested that smaller infarction in MCP-1-deficient mice might be caused by decreased inflammation since induction of IL-6, IL-1 $\beta$ and granulocyte-colony stimulating factor expression and subsequent influx of haematogenous cells was diminished. Additionally similar effects on reduction of infarct size and inflammatory cell infiltration have been observed in mice that were deficient for the MCP-1 receptor (CCR2) [18]. Since our data did not show any influence of ASA + ER-DP on MCP-1 levels, there is so far no argument that lowering of elevated MCP-1 levels might be responsible for amelioration of outcome after treatment with the combination therapy. However, treatment effects by ASA + ER-DP upon this inflammatory marker might have been missed due to study design, since follow-up of MCP-1 determination was not performed before day 8 after stroke.

The temporal course of MCP-1 levels after acute stroke onset shows a rapid increase within $6 \mathrm{~h}$ and a decrease thereafter [3]. In EARLY those patients with high baseline MCP-1 levels show a benefit from early treatment with ASA + ER-DP (Figure 1). High baseline MCP-1 levels in patients might identify a time point after acute stroke at which anti-inflammatory treatment is still effective. This hypothesis, however, cannot be proven since exact time of blood withdrawal was not noted in the case report.

Only further more detailed investigations will be able to shed light as to whether lowering of MCP-1 within the first hours after stroke onset further improves outcome. Other anti-inflammatory properties of dipyridamole might well also contribute to the observed improvement [8-13,19]. Pretreatment of cell cultures of human leukocytes and platelets with dipyridamole inhibited the expression of MCP-1 [8]. In brain endothelial cells dipyridamole attenuated MMP-9 levels after exposure to TNF-alpha or oxygen-glucose deprivation [20]. Dipyridamole inhibited the adhesion of neutrophils to vascular endothelium in acute ischemic stroke patients but not in chronic stroke patients or healthy controls [13]. In a rat model of middle cerebral artery occlusion administration of dipyridamole at reperfusion led to neurological improvement and reduction in infarct size. In this model MCP-1 levels in the infarcted hemisphere were not significantly decreased [21].

The interpretation of MCP-1 levels as a potential marker for decision making upon which of the currently available drugs should be used for early secondary stroke prevention is as yet over-conclusive. Importantly, these effects have only been observed in a subgroup of patients as defined by the inclusion criteria for EARLY [22]. But since recent clinical trials in acute stroke treatment besides the 
thrombolysis trials have failed to show beneficial effects, it has been discussed that due to heterogeneity in stroke patients subgroups need to be defined for treatment approaches. The current data might help to identify those patients that may benefit from a neuroprotective, anti-inflammatory effect of ASA + ER-DP treatment. However, considering that MCP-1 represents only one component of the inflammatory cascade after ischemic stroke the possible role of further mediators has to be elucidated. Moreover, further investigations are required that evaluate the influence of cardiovascular risk factors and comorbidities.

\section{Experimental Section}

\subsection{Patients and Clinical Assessment}

To prove the hypothesis MCP-1 was measured in blood samples from 425 of 543 patients of the EARLY trial taken on admission and day 8 [22]. EARLY was a prospective, randomized, open-label, blinded-endpoint trial, conducted between July 2007, and February 2009, in 46 stroke units in Germany that were certified according to national standards of the German Society of Stroke Medicine. At the start of the trial, patients aged 18 years or more who presented with symptoms of an acute ischemic stroke that caused a measurable neurological deficit (NIHSS score between 5 and 20) were eligible. Patients had to be randomized and treated within $24 \mathrm{~h}$ of stroke symptom onset. Because of slow recruitment during the first 4 months of the trial the inclusion criteria was modified to include patients with minor stroke (NIHSS score $\leq 20$ ). Patients with intracranial haemorrhage identified on CT and those eligible for thrombolysis therapy were excluded. Further exclusion criteria are defined by the EARLY trial protocol [22].

\subsection{Protocol Approval, Registration and Patient Consents}

Patients gave written informed consent before participation in the trial. If patients were unable to sign the informed consent form they had to give verbal informed consent. The protocol was approved by the local institutional review boards and independent ethics committees. The trial was conducted in accordance with the Declaration of Helsinki and the Guideline for Good Clinical Practice [23,24]. Clinical Trial Registration: NCT00562588.

\subsection{Procedures}

Patients were randomly assigned (1:1) either to $25 \mathrm{mg}$ ASA plus $200 \mathrm{mg}$ extended-release dipyridamole orally twice daily within $24 \mathrm{~h}$ of symptom onset (early initiation) for 90 days, or to $100 \mathrm{mg}$ ASA orally once daily for the first 7 days and $25 \mathrm{mg}$ ASA plus $200 \mathrm{mg}$ extended-release dipyridamole orally twice daily from day 8 to day 90 (late initiation). If necessary, study drugs were given via a nasogastric tube. Patients were allowed pre-treatment with antithrombotic agents before hospital admission and at hospital admission.

Tele-mRS was assessed at the Municipal Hospital Munich-Harlaching at days 8 and 90 by an independent expert as described for the EARLY Trial. Patients who died during the study period were assigned a mRS score of 6 . 


\subsection{Measurement of $M C P-1$}

MCP-1 was measured from peripheral venous blood samples (EDTA-plasma) taken from 425 patients on admission and day 8. MCP-1 was determined in EDTA-samples by enzyme-linked immunoabsorbent assay (ELISA) kits (R \& D Systems). The interassay coefficient of variation was shown to be $7.0 \%$. The intra-assay coefficient of variation was shown to be $7.8 \%$.

\subsection{Statistical Analysis}

Statistical analysis was performed using the SAS statistical package, ( $S A S$, version 8.2; SAS Institute: Cary, NC, USA, 2001). A total of 425 patients was included. Patients were stratified by clinical symptoms at baseline and day 8 using the NIHSS (minor symptoms: NIHSS 0 , mild to moderate symptoms: NIHSS 1-5, severe symptoms: NIHSS $>5$ ). Baseline values as well as changes within 8 days of MCP-1 were separated into quartiles. In a first model the predictive value of clinical symptoms at day 8 (minor, mild to moderate, severe) and changes in MCP-1 for the outcome at day 90 was analysed. A second analysis investigated the probability of a favorable outcome at day 90 (mRS 0 or 1) in dependency of baseline MCP-1 quartiles and treatment, adjusted for NIHSS at baseline. Both analyses were performed using logistic regression. The EARLY trial is registered, number NCT00562588.

\section{Conclusions}

In conclusion, the change in MCP-1 levels did not differ in patients treated within $24 \mathrm{~h}$ with ASA + ER-DP in comparison to treatment with ASA alone. However, patients with MCP-1 levels in the highest quartile at baseline showed improved outcome at 90 days if treated with ASA + ER-DP in comparison to treatment with ASA alone despite similar stroke severity at baseline. Further clinical studies are warranted to investigate the role of MCP-1 as a potential marker for deciding on early antithrombotic therapy.

\section{Conflict of Interest}

For H.W., there is no conflict of interest. R.D. has received consulting fees, honoraria, and support for travel to meetings for the study, fees for participation in review activities, payment for development of educational presentations, and travel expenses from Boehringer Ingelheim. H.S. is and W.G.E. has been employees of Boehringer Ingelheim Pharma GmbH and Co. KG. A.S. has received consulting fees, honoraria, support for travel to meetings for the study, fees for participation in review activities, and board membership fees from Boehringer Ingelheim, and honoraria from Sanofi-Aventis. R.L. has received consulting fees, and honoraria from Boehringer Ingelheim. K.W. has received consulting fees and support for travel to meetings for the study from Boehringer Ingelheim.

The study was sponsored by Boehringer Ingelheim. The sponsor was involved in the study design, data collection, data analysis, and interpretation of the data. The sponsor was not involved in the decision to submit the paper for publication. All authors had complete access to the trial data and had responsibility for the final statistical analysis and interpretation of the results. All authors vouch for the 
accuracy and completeness of the data and analyses. The corresponding author was responsible for the decision to submit the paper for publication.

\section{Acknowledgments}

The authors thank Frank Dsiosa, Klaus Burfeind and Bernadette Lüns for excellent technical assistance.

\section{References}

1. Mergenthaler, P.; Dirnagl, U.; Meisel, A. Pathophysiology of stroke: Lessons from animal models. Metab. Brain Dis. 2004, 19, 151-167.

2. Rodríguez-Yáñez, M.; Castillo, J. Role of inflammatory markers in brain ischemia. Curr. Opin. Neurol. 2008, 21, 353-357.

3. Worthmann, H.; Tryc, A.B.; Goldbecker, A.; Ma, Y.T.; Tountopoulou, A.; Hahn, A.; Dengler, R.; Lichtinghagen, R.; Weissenborn, K. The temporal profile of inflammatory markers and mediators in blood after acute ischemic stroke differs depending on stroke outcome. Cerebrovasc. Dis. 2010, 30, 85-92.

4. Welsh, P.; Barber, M.; Langhorne, P.; Rumley, A.; Lowe, G.D.; Stott, D.J. Associations of inflammatory biomarkers with poor outcome in acute ischaemic stroke. Cerebrovasc. Dis. 2009, 27, 247-253.

5. Foerch, C.; Montaner, J.; Furie, K.L.; Ning, M.M.; Lo, E.H. Invited article: searching for oracles? Blood biomarkers in acute stroke. Neurology 2009, 73, 393-399.

6. Chen, Y.; Hallenbeck, J.M.; Ruetzler, C.; Bol, D.; Thomas, K.; Berman, N.E.; Vogel, S.N. Overexpression of monocyte chemoattractant protein 1 in the brain exacerbates ischemic brain injury and is associated with recruitment of inflammatory cells. J. Cereb. Blood Flow Metab. 2003, 23, 748-755.

7. Justicia, C.; Panes, J.; Sole, S.; Cervera, A.; Deulofeu, R.; Chamorro, A.; Planas, A.M. Neutrophil infiltration increases matrix metalloproteinase- 9 in the ischemic brain after occlusion/reperfusion of the middle cerebral artery in rats. J. Cereb. Blood Flow Metab. 2003, 23, 1430-1440.

8. Weyrich, A.S.; Denis, M.M.; Kuhlmann-Eyre, J.R.; Spencer, E.D.; Dixon, D.A.; Marathe, G.K.; McIntyre, T.M.; Zimmerman, G.A.; Prescott, S.M. Dipyridamole selectively inhibits inflammatory gene expression in platelet-monocyte aggregates. Circulation 2005, 111, 633-642.

9. Suzuki, S.; Sugai, K.; Sato, H.; Sakatume, M.; Arakawa, M. Inhibition of active oxygen generation by dipyridamole in human polymorphonuclear leukocytes. Eur. J. Pharmacol. 1992, 227, 395-401.

10. Chakrabarti, S.; Vitseva, O.; Iyu, D.; Varghese, S.; Freedman, J.E. The effect of dipyridamole on vascular cell-derived reactive oxygen species. J. Pharmacol. Exp. Ther. 2005, 315, 494-500.

11. Chakrabarti, S.; Freedman, J.E. Dipyridamole, cerebrovascular disease, and the vasculature. Vasc. Pharmacol. 2008, 48, 143-149.

12. Al-Bahrani, A.; Taha, S.; Shaath, H.; Bakhiet, M. TNF-alpha and IL-8 in acute stroke and the modulation of these cytokines by antiplatelet agents. Curr. Neurovasc. Res. 2007, 4, 31-37. 
13. Hallevi, H.; Hazan-Halevy, I.; Paran, E. Modification of neutrophil adhesion to human endothelial cell line in acute ischemic stroke by dipyridamole and candesartan. Eur. J. Neurol. 2007, 14, 1002-1007.

14. Offner, H.; Subramanian, S.; Parker, S.M.; Afentoulis, M.E.; Vandenbark, A.A.; Hurn, P.D. Experimental stroke induces massive, rapid activation of the peripheral immune system. J. Cereb. Blood Flow Metab. 2006, 26, 654-665.

15. Conductier, G.; Blondeau, N.; Guyon, A.; Nahon, J.L.; Rovère, C. The role of monocyte chemoattractant protein MCP1/CCL2 in neuroinflammatory diseases. J. Neuroimmunol. 2010, 224, 93-100.

16. Hughes, P.M.; Allegrini, P.R.; Rudin, M.; Perry, V.H.; Mir, A.K.; Wiessner, C. Monocyte chemoattractant protein-1 deficiency is protective in a murine stroke model. J. Cereb. Blood Flow Metab. 2002, 22, 308-317.

17. Strecker, J.K.; Minnerup, J.; Gess, B.; Ringelstein, E.B.; Schäbitz, W.R.; Schilling, M. Monocyte chemoattractant protein-1-deficiency impairs the expression of IL-6, IL-1 $\beta$ and G-CSF after transient focal ischemia in mice. PLoS One 2011, 6, e25863.

18. Dimitrijevic, O.B.; Stamatovic, S.M.; Keep, R.F.; Andjelkovic, A.V. Absence of the chemokine receptor CCR2 protects against cerebral ischemia/reperfusion injury in mice. Stroke 2007, 38, $1345-1353$.

19. Kim, H.H.; Liao, J.K. Translational therapeutics of dipyridamole. Arterioscler. Thromb. Vasc. Biol. 2008, 28, s39-s42.

20. Guo, S.; Stins, M.; Ning, M.; Lo, E.H. Amelioration of inflammation and cytotoxicity by dipyridamole in brain endothelial cells. Cerebrovasc. Dis. 2010, 30, 290-296.

21. García-Bonilla, L.; Sosti, V.; Campos, M.; Penalba, A.; Boada, C.; Sumalla, M.; Hernández-Guillamon, M.; Rosell, A.; Montaner, J. Effects of acute post-treatment with dipyridamole in a rat model of focal cerebral ischemia. Brain Res. 2011, 1373, 211-220.

22. Dengler, R.; Diener, H.C.; Schwartz, A.; Grond, M.; Schumacher, H.; Machnig, T.; Eschenfelder, C.C.; Leonard, J.; Weissenborn, K.; Kastrup, A.; et al. EARLY Investigators. Early treatment with aspirin plus extended-release dipyridamole for transient ischaemic attack or ischaemic stroke within $24 \mathrm{~h}$ of symptom onset (EARLY trial): A randomised, open-label, blinded-endpoint trial. Lancet Neurol. 2010, 9, 159-166.

23. Declaration of Helsinki. Ethical Principles for Medical Research Involving Human Subjects. In Proceeding of 48th World Medical Association General Assembly, Somerset West, South Africa, 22-26 October 1996. Available online: http:/www.wma.net/en/30publications/10policies/ b3/index.html (accessed on 1 May 2012).

24. Guideline for Good Clinical Practice E6(R1). International Conference on Harmonisation Harmonised Tripartite Guideline, 1996. Available online: http://www.ich.org/fileadmin/Public Web_Site/ICH_Products/Guidelines/Efficacy/E6_R1/Step4/E6_R1_Guideline.pdf (accessed on 1 May 2012).

(C) 2012 by the authors; licensee MDPI, Basel, Switzerland. This article is an open access article distributed under the terms and conditions of the Creative Commons Attribution license (http://creativecommons.org/licenses/by/3.0/). 